\title{
AID for reprogramming
}

\author{
Suneet Agarwal $^{1}$, George Q Daley ${ }^{1}$ \\ ${ }^{I}$ Division of Hematology/Oncology, Children's Hospital Boston; Pediatric Oncology, Dana Farber Cancer Institute; Manton Center \\ for Orphan Disease Research; Harvard Stem Cell Institute; Division of Hematology, Brigham and Women's Hospital; Department of \\ Biological Chemistry and Molecular Pharmacology, Harvard Medical School; Howard Hughes Medical Institute, Children's Hospital \\ Boston, 1 Blackfan Circle, Boston, MA 02115, USA \\ Cell Research (2010) 20:253-255. doi: 10.1038/cr.2010.30; published online 1 March 2010
}

Five decades of nuclear transfer (NT) experiments have established a key principle in developmental genetics - despite vast functional differences, virtually all of the cells in an adult organism maintain an identical genome [1]. Studies in several species have shown that the nucleus of a differentiated cell can be reprogrammed by exposure to egg cytoplasm, which re-initiates an embryonic genetic program in the transferred genome and permits the development of an identical adult organism (i.e. cloning). The demonstration that one adult cell can give rise to any other cell type has fueled tremendous interest in therapeutic applications, wherein a patient's own healthy cells would be reprogrammed to replace other cell types that have been damaged by disease or age. However, despite a half century of NT experiments in several species, the process by which genomes are reprogrammed remains largely unknown, in large part due to the inefficiency of NT and experimental limitations in manipulating the egg cytoplasm. Other, more tractable methods exist to reprogram cells from an adult to embryonic (or pluripotent) phenotype, including cell fusion [2] and direct reprogramming to create induced pluripotent stem (iPS) cells [3]. In a recent report, Bhutani et

Correspondence: George Q Daley

E-mail: George.Daley@childrens.harvard.edu al. [4] begin to shed some light into the "black box" of reprogramming (Figure 1). By demonstrating the rapid and efficient induction of pluripotencyassociated genes in human fibroblasts after fusion to mouse embryonic stem (ES) cells, and the perturbation of this induction when a candidate factor (Activation-induced Cytidine Deaminase, or AID) is disrupted, the authors pro-

vide new insights into the mechanisms regulating the path to pluripotency. As importantly, in doing so, they bolster emerging evidence that AID may be involved in the elusive process of active DNA demethylation.

To assay for reprogramming towards pluripotency, Bhutani et al. used polyethylene glycol (PEG)-mediated fusion of somatic cells and ES cells to create

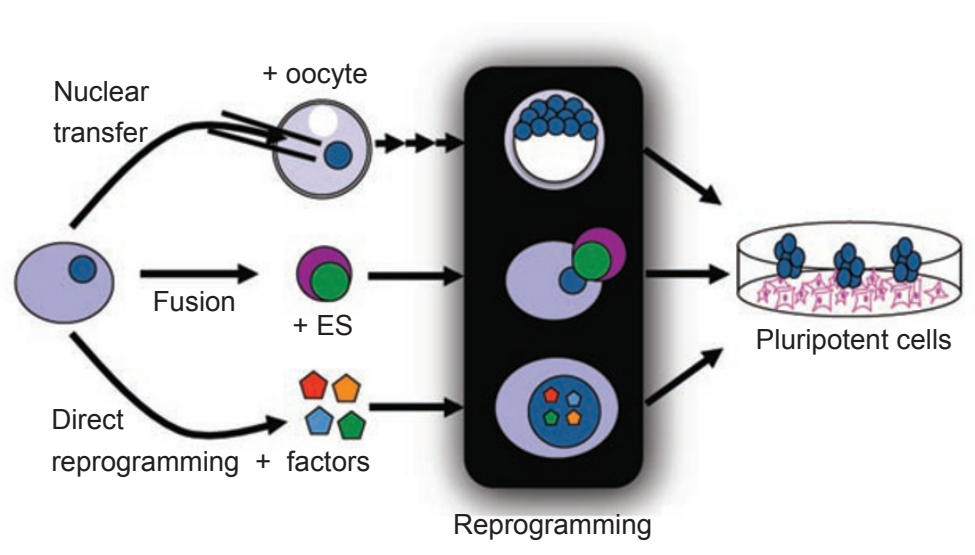

Figure 1 The black box of reprogramming. Despite the innovation of multiple methods to convert a somatic cell to a pluripotent phenotype over the past 50 years, the mechanisms remain largely unknown. Reprogramming methods: (1) Nuclear transfer: injection of a mammalian somatic cell nucleus into an enucleated oocyte will re-initiate the zygotic gene program in the transferred nucleus and permit embryonic development to the blastocyst stage (or later), from which pluripotent cells can be derived. (2) Cell fusion: after fusion of somatic cells with embryonic stem (ES) cells, the genetic program of the ES cell dominates in resulting heterokaryons, with pluripotent gene expression and epigenetic patterns evident in the somatic cell nucleus. (3) Direct reprogramming: introduction of defined transcription factors (e.g. OCT4, SOX2, KLF4, MYC) into somatic cells followed by selection under ES cell culture conditions yields induced pluripotent stem (iPS) cells, with morphological and functional features indistinguishable from blastocyst-derived ES cells. 
heterokaryons, in which the genetic program of the pluripotent cell has been found to dominate [2]. Because they used interspecies cell fusion, the authors were able to distinguish new gene expression from the human nucleus as opposed to the mouse nucleus. Compared to human fetal lung fibroblasts that were co-cultured (rather than fused) with mouse ES cells, interspecies heterokaryons showed a modest 10 -fold induction of the pluripotency-associated genes $O C T 4$ and $N A N O G$ after 3 days. To assess the efficiency of OCT4 and NANOG induction, the authors sorted single heterokaryons and found that $70 \%$ of the fused cells expressed both genes. Thus, in contrast to iPS cell generation, reprogramming of the human genome (as measured by the induction of pluripotency-associated genes) by fusion with mouse ES cells is rapid, and provided the authors with an assay to investigate the molecular mechanisms of OCT4 and NANOG gene activation.

$\mathrm{CpG}$ methylation is an epigenetic mark associated with heterochromatin and transcriptional silencing in mammalian DNA. Using bisulfite conversion followed by cloning and sequencing of PCR amplicons, the authors investigated methylation changes at the endogenous human OCT4 and NANOG promoters following cell fusion. In correlation with gene expression, fibroblasts possessed heavily methylated OCT4 and NANOG loci, whereas day 3 heterokaryons showed frequent demethylation at both promoters. This observation allowed the authors to approach the long-standing question of how mammalian DNA is demethylated. CpG demethylation may occur passively by failure of maintenance methylation following DNA replication, or actively by the replication-independent modification and/or removal of methylcytidine from DNA [5]. However, because the mechanisms of active demethylation have been extremely difficult to define, the existence of this pathway remains a subject of considerable controversy
[6]. In this context, Bhutani et al. made the remarkable observation that despite demethylation of the $O C T 4$ and NANOG promoters, $>94 \%$ of heterokaryons did not undergo cell division by 3 days after fusion, as judged by BrdU incorporation and staining for the proliferationassociated antigen Ki67. These observations suggest that the human OCT4 and $N A N O G$ promoters are subject to active demethylation after fusion with mouse ES cells, and that cell division is not essential for reprogramming of these loci. The authors next investigated one of several proposed pathways for active demethylation - deamination of methylcytidine by AID/APOBEC factors, followed by repair of the resulting thymidine:guanine mismatch with unmethylated cytidine [5]. They focused on AID, best known for its role in class switch recombination and somatic hypermutation of immunoglobulin genes in B cells [7], because of its presence in pluripotent cells [8] and proposed involvement in active DNA demethylation in zebrafish post fertilization [9]. After siRNA-mediated disruption of AID in both fusion partners, induction of $O C T 4$ and $N A N O G$ gene expression in heterokaryons was severely abrogated, which correlated with a lack of OCT4 and $N A N O G$ promoter demethylation 3 days post-fusion. This effect could be rescued by ectopic expression of human AID protein in the mouse ES cells prior to fusion. These results implicate AID in the demethylation of the OCT4 and $N A N O G$ loci after cell fusion, and support the model of indirect active demethylation of mammalian DNA by a mechanism involving cytidine deaminases. The authors finally showed that AID proteins were associated with the promoters of inactive but not active genes in human fibroblasts and mouse ES cells, suggesting that AID proteins directly bind methylcytidine at silent genetic loci and stand poised to modify these residues in response to activating signals.

Collectively, the results of Bhutani et al. demonstrate how cell fusion can be used to dissect the molecular events underlying developmental changes in cell fate. Several important questions remain: (1) Are the same mechanisms at work during reprogramming by NT or in the generation of iPS cells? The possibility is favored by the observations of active demethylation of the paternal genome after natural fertilization [10], and the facilitation of iPS cell generation using agents that interfere with maintenance of DNA methylation [11]. Moreover, AID has recently been implicated in epigenetic reprogramming via erasure of DNA methylation in primordial germ cells [12]. The results of Bhutani et al. suggest that AID function is not replaceable by other cytidine deaminase family members. Therefore, it would be interesting to compare the efficiency of NT and direct reprogramming using cells from wild-type versus AID null mice, and to assess the effects of $A I D$ disruption on human iPS cell generation, either through AID knockdown or using cells from patients with Hyper-IgM Syndrome 2 caused by AID mutations. (2) What are the mechanisms activating AID during reprogramming? The observation by Bhutani et al. that AID binds to the OCT4 and NANOG promoters in fibroblasts, where the loci remain methylated, implies that the presence of AID is not sufficient for demethylation. Factors either resident in mouse ES cells or induced in the heterokaryons must therefore be invoked to explain AID-mediated activation of demethylation at these loci after fusion. (3) What are the other steps and components in a proposed pathway leading from deamination of methylcytidine to replacement by unmethylated cytidine? In showing that candidate factors can be manipulated to perturb gene induction and DNA demethylation at specific loci in cell fusion assays, the study by Bhutani et al. provides an experimental system to illuminate not just one but two black boxes of developmental genetics. 


\section{References}

1. Gurdon JB, Byrne JA. The first halfcentury of nuclear transplantation. Proc Natl Acad Sci USA 2003; 100:80488052.

2. Miller RA, Ruddle FH. Pluripotent teratocarcinoma-thymus somatic cell hybrids. Cell 1976; 9:45-55.

3. Takahashi K, Yamanaka S. Induction of pluripotent stem cells from mouse embryonic and adult fibroblast cultures by defined factors. Cell 2006; 126:663676.

4. Bhutani N, Brady JJ, Damian M, Sacco A, Corbel SY, Blau HM. Reprogramming towards pluripotency requires AID-dependent DNA demethylation. Nature 2009 Dec 21. doi:10.1038/na- ture 08752

5. Morgan HD, Santos F, Green K, Dean W, Reik W. Epigenetic reprogramming in mammals. Hum Mol Genet 2005; 14 Spec:R47-R58.

6. Ooi SK, Bestor TH. The colorful history of active DNA demethylation. Cell 2008; 133:1145-1148.

7. Delker RK, Fugmann SD, Papavasiliou FN. A coming-of-age story: activationinduced cytidine deaminase turns 10 . Nat Immunol 2009; 10:1147-1153.

8. Morgan HD, Dean W, Coker HA, Reik W, Petersen-Mahrt SK. Activationinduced cytidine deaminase deaminates 5-methylcytosine in DNA and is expressed in pluripotent tissues: implications for epigenetic reprogramming. $J$ Biol Chem 2004; 279:52353-52360.
9. Rai K, Huggins IJ, James SR, Karpf AR, Jones DA, Cairns BR. DNA demethylation in zebrafish involves the coupling of a deaminase, a glycosylase, and gadd 45 . Cell 2008; 135:1201-1212.

10. Mayer W, Niveleau A, Walter J, Fundele R, Haaf T. Demethylation of the zygotic paternal genome. Nature 2000; 403:501502.

11. Meissner A, Mikkelsen TS, Gu H, et al. Genome-scale DNA methylation maps of pluripotent and differentiated cells. Nature 2008; 454:766-770.

12. Popp C, Dean W, Feng S, et al. Genomewide erasure of DNA methylation in mouse primordial germ cells is affected by AID deficiency. Nature 2010 Jan 22. doi:10.1038/nature08829 